\title{
Un nouveau point de départ pour l'histoire de l'Atlantique central
}

\author{
Mohamed Sahabi ${ }^{\mathrm{a},{ }^{*}}$, Daniel Aslanian ${ }^{\mathrm{b}}$ and Jean-Louis Olivet ${ }^{\mathrm{b}}$ \\ a Laboratoire Géosciences marines, faculté des sciences d'El Jadida. BP 20, 24000, El Jadida, Maroc \\ ${ }^{\mathrm{b}}$ Département de recherches océanographiques-Géosciences marines, Ifremer, centre de Brest, 29280 , \\ Plouzané, France \\ *: Corresponding author : Laboratoire Géosciences marines, Faculté des Sciences d'El Jadida. B.P. 20, 24000, El \\ Jadida, Maroc. Tél. 212.23.34.23.25 ; Fax : 212.23.34.21.87 ; email : msahabi@ucd.ac.ma
}

\begin{abstract}
The first oceanic crust in the central Atlantic is usually thought to have a Middle Jurassic age. The new interpretation of the two key parameters, the African homologue of the East Coast Magnetic Anomaly and the situation of the Triassic salt basin of Morocco and Novia Scotia, shows that this age was underestimated by about $20 \mathrm{Ma}$. In our kinematic reconstruction, the first oceanic crust begins at the Late Sinemurian. This difference in age is crucial for the evolution of those margins and we discuss here its consequences.
\end{abstract}

Résumé: La première croûte océanique de l'Atlantique central est le plus souvent attribuée au Jurassique moyen. Notre reconstruction la situe à la fin du Sinémurien, soit environ 20 Ma plus tôt, différence capitale pour la modélisation des marges américaine et africaine. Cette révision, qui met fin à nombre de contradictions, est fondée sur la réinterprétation de deux éléments clés : l'équivalent africain de la East Coast Magnetic Anomaly, d'une part, l'extension des bassins à évaporites triasicoliasiques du Maroc et de Nouvelle-Écosse, d'autre part. L'article est consacré à cette réinterprétation et à ses conséquences en termes d'âge.

Keywords: Central Atlantic, ECMA, Salt Basin, Cinematic

Mots-clés : Atlantique central; anomalie magnétique; bassin à évaporites; cinématique 


\section{Abridged English version}

The African and American margins of the central Atlantic Ocean have a major historical and geological importance: 1- American margins were the place of the most intense geological and geophysical exploration and synthesis related to continental margins (most concepts were defined or tested there); 2- in contrast to the North Atlantic which has a complex rifting history, breakup in the central Atlantic seems to occur in a single phase, at the Trias-Lias boundary, allowing for much easier modelling; 3- contrary to the South and North Atlantic, intracontinental deformation is not so crucial and does not complicate the initial reconstruction; 4- the Central Atlantic is the very place (with the exception of the Alpes) to "read" the breakup history of Pangea and the beginning of the closure of the Thetys Ocean.

Since 1986, the breakup story of this region has been largely based on the interpretation of Klitgord \& Schouten, which did not take into account the African margin and its magnetic anomalies and salt basins. We propose here a new reconstruction with a new interpretation of these African data.

The American margin (Fig 1) is characterised by a strong, continuous magnetic anomaly, the East Coast Magnetic Anomaly (ECMA), and a second magnetic anomaly, the Blake Spur Magnetic Anomaly (BSMA). The ECMA, fringed throughout by a salt basin, is thought to represent the continentaloceanic boundary. In the north nevertheless, the salt runs largely over the ECMA (Fig $1 \& 2$ ).

The African homologue of the ECMA, the West African Coast Magnetic Anomaly (WACMA), is not so well defined due to its weaker amplitude and to a lack of data (Fig 3). Our interpretation of this anomaly is based on all available magnetic data and the following two important points : the similarity in shape of both magnetic anomalies and the position of the Mauritanian salt basin. In contrary to Klitgord \& Schouten (1986), who considered the anomaly $\mathrm{S}$ in the Morocco Basin as the homologue of the BSMA (Lower Callovian), this anomaly represents the northern part of the WACMA in our interpretation. This modification has important consequences on the age of the fit which had been supposed to be Middle Jurassic, or more precisely $175 \mathrm{Ma}$, by simple extrapolation of the spreading rate between the anomalies M25 and BS to the fit.
In figure 6 (which is derived from the fit shown in figure 5), we present a "slightly opened fit" in order to illustrate the perfect homology of the two magnetic anomalies. Note that here, as well as in figure 5, the Moroccan Meseta is slightly disconnected from the west-African craton in order to take into account the younger atlasic compression and to improve the fit.

This new interpretation of the WACMA allows us to propose a new reconstruction at $195 \mathrm{Ma}$ (top of Sinemurian) of the Central Atlantic (Fig 5) which gives a more coherent position of ECMA relative to the African structures and enables us to date it precisely: it juxtaposes the ECMA and the WACMA and places side by side the large salt basins of Morocco and Nova Scotia, as well as those of Mauritania and Carolina. The outflowing salt, in the northern part of the area, is interpreted as a large post-rifting slide, as shown on the figure 2. Both the ECMA and WACMA coincide with the limit of the salt basin. This fit of the two magnetic anomalies is therefore the paleo-reconstruction of the lower-liasic salt basin, at its maximum extension. The age of these magnetic anomalies is also connected to the end of the salt deposition, i.e $195 \mathrm{Ma}$ (Late Sinemurian), $20 \mathrm{Ma}$ older than previously thought. Moreover, this age is in good agreement with the age of the volcanic activity on both sides of the Atlantic ocean (Lower Lias, just before the end of the salt deposition).

The BSA, identified and dated on the American plate (lower Callovian), is also situated in an intraoceanic context: during 30 to $40 \mathrm{Ma}$, only a small amount of oceanic crust was created, pointing out to a very slow spreading. The well-described tectonic and magmatic activity of the Middle Jurassic (even maybe since Toarcian) should therefore be distinguished from the rifting activity (Trias-Lias boundary); it seems to coincide with an important acceleration of the African/American and African/European plate movements.

\section{Introduction}

La marge nord américaine de l'Atlantique Central, en regard de l'Afrique de l'Ouest, est celle qui a donné lieu à l'effort d'exploration (par sismique réflexion lourde, réfraction, forages profonds et levés magnétique et gravimétrique) et de synthèse le plus important concernant les marges continentales. Son interprétation gouverne encore à bien des égards celle des autres marges ainsi que des bassins intracontinentaux : n'est-elle pas la marge continentale passive type, «the atlantic type margin » [33] ? La plupart des concepts utilisés aujourd'hui (comme 
« hinge line », « synrift », « breakup », « post rift unconformity » ou " transitional zone») y ont été soit forgés, soit testés avant d'être adoptés par la communauté scientifique. Mais cette importance historique tient aussi à des raisons géologiques précises : premièrement, la rupture continentale complète entre l'Amérique du Nord est l'Afrique paraît s'être déroulée en une seule phase, à cheval sur le Trias et le Lias, au contraire de ce qui s'est produit pour l'Atlantique Nord où la plupart des marges ont enregistré des phases distinctes s'étageant sur une très longue période (du Permo-Trias au Crétacé ou à 1'Eocène): elle se prête donc mieux à la modélisation.

Deuxièmement, la question de la déformation intra-continentale s'y pose moins que dans l'Atlantique Nord et dans 1'Atlantique Sud: les épisodes de déformation qui ont affecté l'Europe dans le premier cas, 1'Afrique Equatoriale et l'Amérique du Sud dans le second, compliquent la question de la «reconstruction initiale» de ces océans, c'est-à-dire de la reconstruction de l'état des plaques à l'époque où se mettait en place le mécanisme de «sea floor spreading». Pour l'Atlantique Central, en toute rigueur, se pose le problème de la Meseta Marocaine, séparée du craton Ouest-Africain par le sillon atlasique; mais l'ouverture de ce dernier, contemporaine de celle de l'Atlantique, ne pose qu'un problème mineur pour les reconstructions. On peut donc considérer que l'évolution des marges de l'Atlantique Central relève d'une cinématique aussi simple que possible.

Troisièmement enfin, sur un autre plan, les marges de 1'Atlantique Central tiennent une place capitale : si l'on considère la rupture de la Pangée, entre Gondwana et Laurasia, comme marquant le début du cycle alpin, elles constituent le meilleur endroit où en étalonner la première étape, indépendamment des Alpes elles-mêmes. Or nous pensons que l'histoire atlantique de cette rupture, principalement fondée jusqu'ici sur l'interprétation de la marge américaine et le modèle cinématique de Klitgord et Schouten [14], doit être reconsidérée. La raison en est principalement que les données de la marge africaine homologue n'ont jamais été suffisamment prises en compte : ainsi, le bassin à évaporites connu au large du Maroc [25, 6, 23] n'a été intégré aux modèles d'ouverture que de manière schématique $[14,8,42]$ ou sous la forme d'une question non résolue [15]. De ce fait découle que le trait géophysique majeur de la marge américaine, la
«East Coast Magnetic Anomaly (ECMA), qui joue un rôle crucial dans toutes les interprétations, n'a jamais pu être ni daté, ni situé de manière cohérente par rapport aux structures de la marge africaine.

Nous proposons ici une reconstruction fondée sur la réinterprétation des deux éléments clé que sont la définition de l'équivalent africain de la "ECMA » d'une part, l'extension des bassins à évaporites triasico-liasiques d'autre part.

\section{Les structures de la marge américaine}

\subsection{La « East Coast Magnetic Anomaly 》}

La fig. 1) indique les principaux traits géophysiques et structuraux de la marge américaine de 1'Atlantique Central. La «East Coast Magnetic Anomaly » (ECMA, [37]) est représentée d'après la carte synthétique de Verhoef et al. [36] : cette anomalie magnétique, de forte amplitude et remarquablement continue, suit de près le bord du plateau continental, sauf au Sud de la Nouvelle Ecosse où elle s'en écarte. Elle reproduit, à $40^{\circ} \mathrm{N}$ et $42^{\circ} \mathrm{N}$, les deux grands décalages que fait la pente continentale. Au Sud, elle se termine à la limite de la Floride où elle oblique vers l'intérieur du continent américain en suivant les structures paléozoïques. On peut distinguer trois segments en fonction de l'allure de l'anomalie : dans le segment méridional, jusque vers $36^{\circ} \mathrm{N}$, et dans le segment septentrional, entre $41^{\circ} \mathrm{N}$ et $44^{\circ} \mathrm{N}, 1^{\prime}$ anomalie présente une structure double tandis que la partie médiane est dominée par une anomalie simple. Pratiquement tous les travaux s'accordent à considérer que la ECMA marque, d'une manière ou d'une autre, la limite entre le continent et océan; d'un autre côté, on ne peut manquer de remarquer l'étroite parenté de direction entre les structures appalachiennes et le tracé de l'anomalie qui, de ce fait, a souvent été interprétée comme la trace de la suture paléozoïque $[19,21]$. Les deux points de vue ne sont pas exclusifs l'un de l'autre. Les études de sismique, quant à elles, concluent toutes à la présence de croûte océanique typique dès sa limite externe $[32,7,12]$.

On a indiqué sur la fig.1, d'après les travaux de synthèse sur le substratum paléozoique $[33,13]$, le tracé de la «hinge line» qui est théoriquement la ligne à partir de laquelle le substratum continental s'approfondit rapidement vers le large en réponse à l'amincissement de la croûte continentale. La plus grande épaisseur sédimentaire se retrouve effectivement comprise, d'une manière générale, entre cette ligne et le bord externe de la ECMA. On 
peut observer la parenté de forme entre l'anomalie magnétique et la « hinge line », les deux structures étant même accolées dans le segment méridional.

La marge américaine est caractérisée par une seconde anomalie magnétique remarquable, la « Blake Spur Magnetic Anomaly » (BSMA) ainsi que par une anomalie plus discrète, un peu antérieure, l'anomalie E [27]. Datée par forage du Callovien [34], et clairement située en domaine océanique, on verra que la BSMA tient aussi une place déterminante dans le problème de la reconstruction initiale.

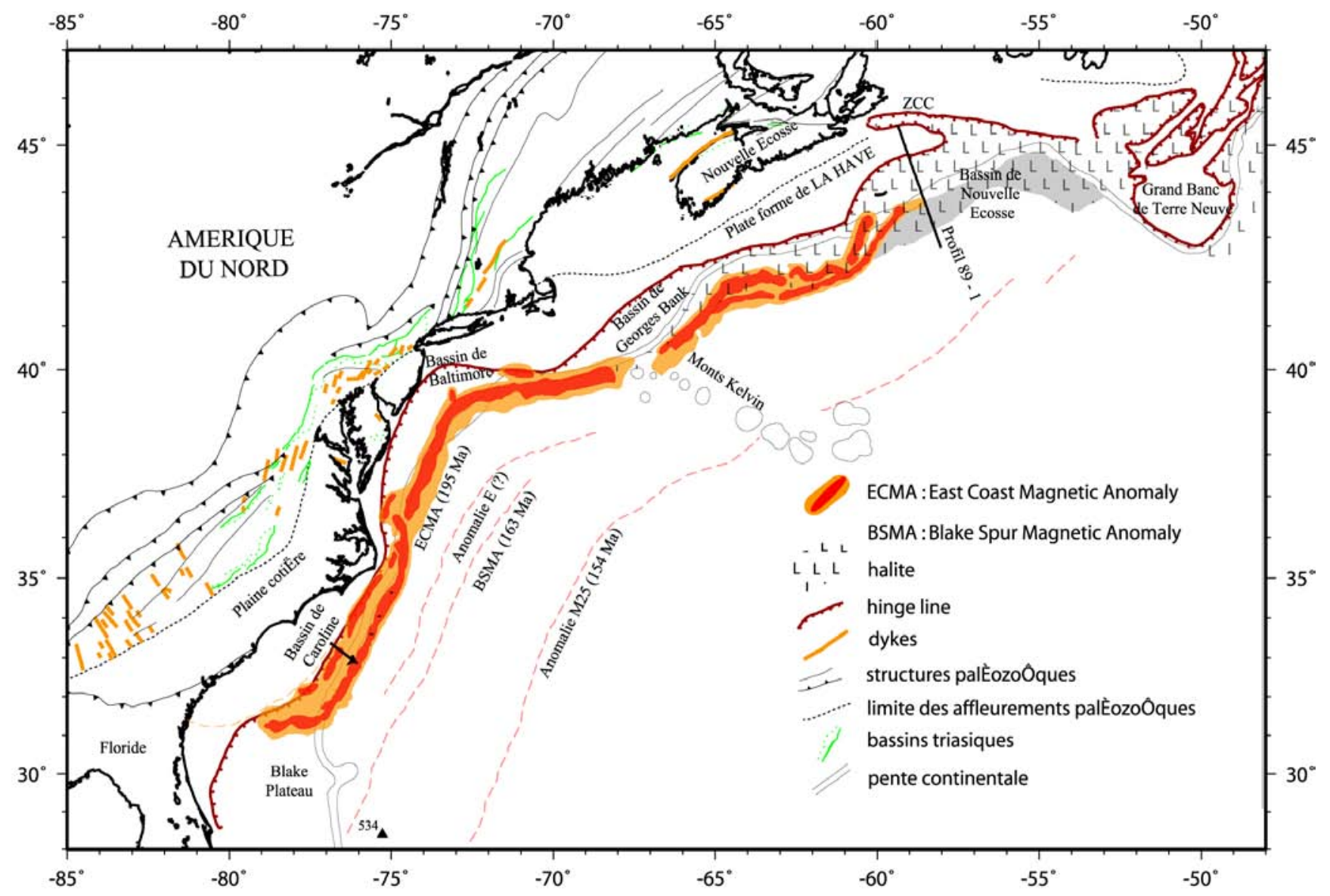

Figure 1 : Carte générale de la marge est américaine. La East Coast Magnetic Anomaly (ECMA) a été représentée en deux tons, en fonction de sa forme et de son amplitude, à partir de la carte de [36]. Noter sa largeur à peu près constante (70-90 $\mathrm{km}$ ), avec une morphologie variable selon les segments. Le bassin de Caroline est situé à l'aplomb de la partie externe de la ECMA. Les autres bassins (Baltimore, Georges Bank et Nouvelle Ecosse) se situent dans des rentrants de la « hinge Line » par rapport à la ECMA. L'âge indiqué (195 Ma) correspond approximativement à la première croûte océanique adjacente à cette anomalie. Les affleurements volcaniques (dykes) reportés d'après [33] appartiennent à la province magmatique (CAMP). La carte montre également la répartition des dépôts de halite avec, en grisé, l'extension du glissement illustré par la coupe de la figure 2 (profil 89-1). L'anomalie E est la première anomalie magnétique notable à partir de la marge. Le forage DSDP 534 qui a permis de dater la Black Spur Magnetic Anomaly (BSMA) est indiqué.

Figure 1: General map of the East-American margin. The East Cost Magnetic Anomaly (ECMA), drawn after the magnetic map of [36], is represented in two colors, depending of its shape and amplitude. Note its constant width (70-90 km) and its variable morphology (double or simple). The Carolina basin is situated straight above the outern limit of the ECMA. Baltimore, Georges Bank and Novia Scotian Basins are delimited by the ECMA and bends of the hinge line. The age indicated (195 Ma) corresponds approximatively to the first oceanic crust. Dykes are from [33] and belong to the Central Atlantic Magmatic Province (CAMP). Black triangle shows the position of the core of DSDP 534, which gave the age of the Black Spur Magnetic Anomaly (BSMA) (154 Ma). 


\subsection{Les bassins à évaporites}

Les évaporites du Trias et du début du Lias sont les sédiments à affinité marine les plus anciens identifiés sur le pourtour de l'Atlantique Central depuis les dépôts carbonifères $[17,38]$. Pautot et al [25] en ont les premiers interprété la présence sur un profil sismique situé au NW de la Meseta marocaine ; imaginant le passage par un stade de type «mer Rouge » à l'origine de l'océan actuel, ils ont postulé l'existence de ce sel tout autour de l'Atlantique Central. Les travaux postérieurs n'ont qu'en partie confirmé cette première interprétation : des bassins à évaporites existent effectivement, mais disjoint les uns des autres, et d'importance très inégale. Comme le montre la fig. 1, le bassin américain de loin le plus important est celui de Nouvelle Ecosse qui trouve son prolongement, décalé par la zone de Chedabucto, dans les bassins du Grand Banc de Terre-Neuve. Les évaporites, datées à partir de forages, appartiennent à la formation ARGO [11,39], qui s'étend du Rhétien à la fin du Sinémurien. Une formation à évaporites plus ancienne (datée du Carnien) existe sur le Grand Banc, mais non sur les marges de l'Atlantique Central proprement dit. On trouve encore, en allant vers le Sud, des évaporites dans la partie profonde du bassin de Georges Bank, mais elles sont absentes du bassin de Baltimore Canyon. On en trouve à nouveau dans un bassin très étroit $(30 \mathrm{~km}$ de largeur sur $300 \mathrm{~km}$ de longueur) à l'aplomb de la ECMA, le bassin de Caroline [3].

Les évaporites de Nouvelle Ecosse sont elles aussi délimitées par la ECMA sur près de $700 \mathrm{~km}$, de $40^{\circ} \mathrm{N}$ à $42^{\circ} 30 \mathrm{~N}$, mais au-delà vers le Nord, elles débordent cette limite de 50 à $70 \mathrm{~km}$ [13]. Cette disposition est problématique puisqu'elle oblige à considérer soit que la ECMA ne correspond pas à la limite océan/continent, soit que cette limite est plus ancienne que les évaporites.

Nous avons repris (fig. 2) l'interprétation d'un profil de sismique réflexion situé dans le domaine où les manifestations halocinétiques sont observées au delà de la ECMA.

Keen et Potter [12], qui ont publié ce profil, attribuent à la fin du Jurassique l'horizon au-dessus duquel s'observent les derniers diapirs en allant vers le large. On voit que la couche responsable des phénomènes diapiriques est également à l'origine d'un décollement de la couverture au pied de la pente continentale. Notre interprétation souligne le fait que le niveau de décollement affecte des niveaux de plus en plus anciens en allant vers le continent. Nous pensons donc que si les évaporites de la formation ARGO sont bien à l'origine des manifestations diapiriques et du glissement de la couverture, comme l'ont admis tous les auteurs, c'est à condition d'être sorties de leur bassin d'origine. A notre connaissance ce fait n'avait jamais été relevé.

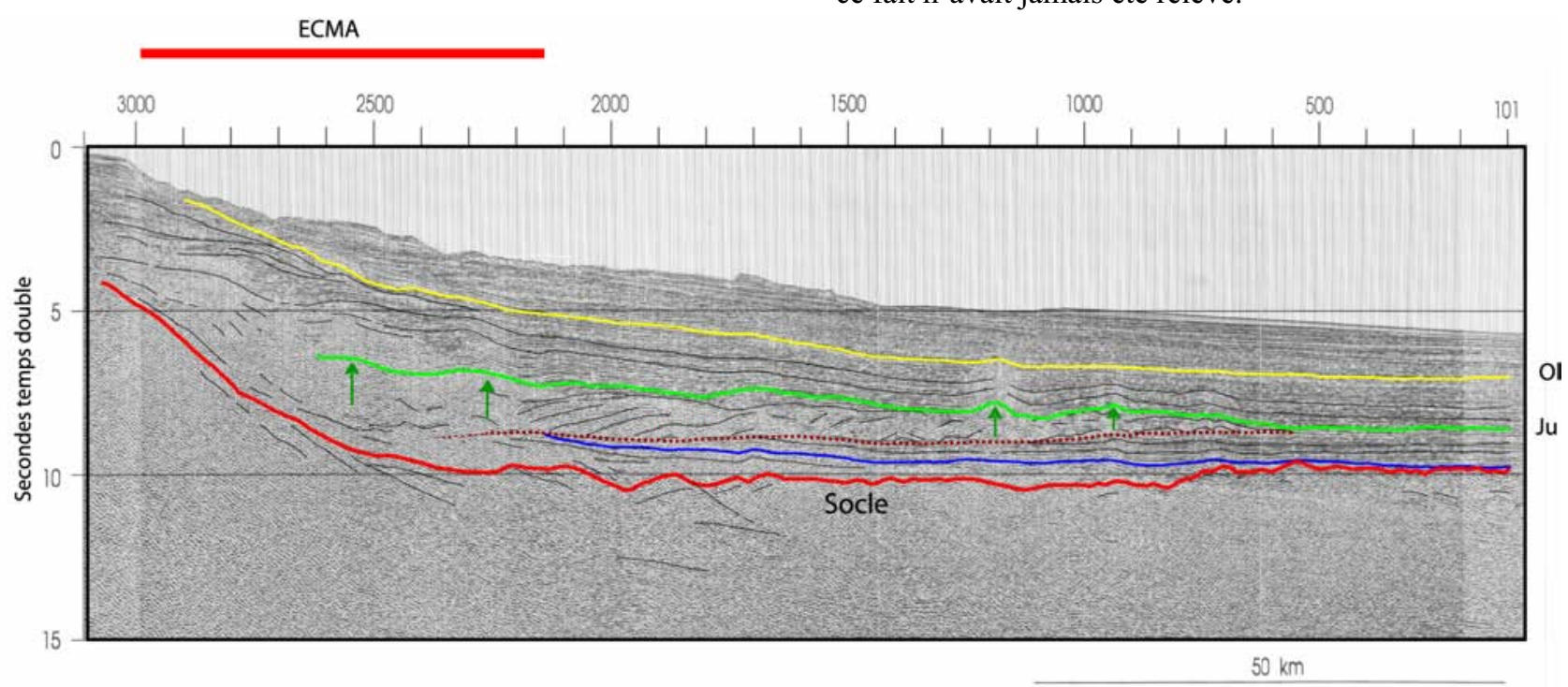

Figure2 : Le profil 89-1 [12] et son interprétation. La ECMA est positionnée au dessus du profil. Les flèches indiquent des diapirs. Le réflecteur en pointillé correspond au mur de la couche origine des diapirs, au delà de ECMA. Il rejoint vers le large, l'horizon vert daté de la fin du Jurassique. L'horizon jaune est Oligocène; l'horizon bleu, sous les évaporites, est intra-Jurassique (d'après[39]). Noter les figures de glissement entre le réflecteur en pointillé et le réflecteur vert.

Profile 89-1 [12] and its interpretation. The ECMA is located above the profile. Arrows indicate the diapirs. Dashed reflector corresponds to the base of the original layer of the diapirs, beyond the ECMA. Green reflector: Late Jurassic ; yellow reflector: Oligocene ; blue reflector under the salt: intra-Jurassic (after [39]). Note the sliding features between the dashed and the green reflectors. 
Cet accident peut avoir son origine dans la phase tectonique de la base du Crétacé («Avallon Uplift» du Grand Banc de Terre-Neuve) qui, selon Wade et Mac Lean [39], affecte aussi la Nouvelle Ecosse. La ECMA indique donc bien, là où elle existe, la limite $\mathrm{du}$ bassin à évaporites. Ce lien entre anomalie magnétique et limite du bassin à évaporites se retrouve du côté africain.

\section{Les structures de la marge africaine}

\subsection{L'anomalie ouest-africaine (fig. 3)}

Si la ECMA signe la limite entre océan et continent, la marge africaine doit nécessairement présenter une anomalie similaire. Au Nord de $26^{\circ} \mathrm{N}$, les travaux à la mer, allemands principalement [38, 28], ont été suffisamment nombreux pour permettre une comparaison directe avec le côté américain. Les synthèses publiées par Verhoef et al [36] et Roeser et al [28] montrent, entre $26^{\circ} 30$ à $32^{\circ} 30 \mathrm{~N}$, de part et d'autre des Canaries, une anomalie (dénommée S1) très semblable par sa forme et sa situation à la ECMA, avec toutefois deux différences: une amplitude nettement moindre et une prolongation vers le Nord (de $32^{\circ} 30^{\prime} \mathrm{N}$ jusque vers $34^{\circ} 30^{\prime} \mathrm{N}$ ) sans équivalent du côté américain ; nous appellerons $\mathrm{S}$ ' cette portion de l'anomalie S1 dépourvue d'élément de symétrie. Plus encore que son homologue canadienne, l'anomalie $\mathrm{S} 1$ tend à s'écarter du bord du plateau continental en allant vers le Nord. Une autre anomalie, S3, double en partie 1'anomalie S1 entre $29^{\circ} \mathrm{N}$ et $32^{\circ} \mathrm{N}$, lui donnant une allure comparable à celle de la ECMA sur la marge canadienne.

L'anomalie S1 semble se terminer au Sud vers $26^{\circ} 30 \mathrm{~N}$, en même temps qu'elle rencontre la côte du Sahara Marocain. Un levé magnétique à terre décrit par Queroll in [41] montre en effet qu'elle ne s'y trouve pas. On retrouve par contre vers le Sud une anomalie côtière assimilable à l'anomalie $\mathrm{S}$ à partir de $25^{\circ} 30 \mathrm{~N}$. Mise progressivement en évidence par différents auteurs [29, 5, 35], elle s'observe sur de nombreux profils jusqu'à la latitude du cap Mirik, au voisinage de la côte Mauritanienne. Plus au Sud encore, Liger [16] a décrit à terre et interprété une anomalie reconnue à partir d'un levé aéromagnétique entre $15^{\circ} \mathrm{N}$ et $18^{\circ} \mathrm{N}$. Cette anomalie est remarquablement rectiligne sur plus de $300 \mathrm{~km}$, tout comme le segment de la ECMA qui lui fait face. Reprenant l'ensemble des données, Roussel et Liger [30] et Olivet et al [23] ont estimé que cette anomalie du Sénégal pouvait être considérée comme la terminaison vers le sud de l'anomalie $\mathrm{S}$ et que

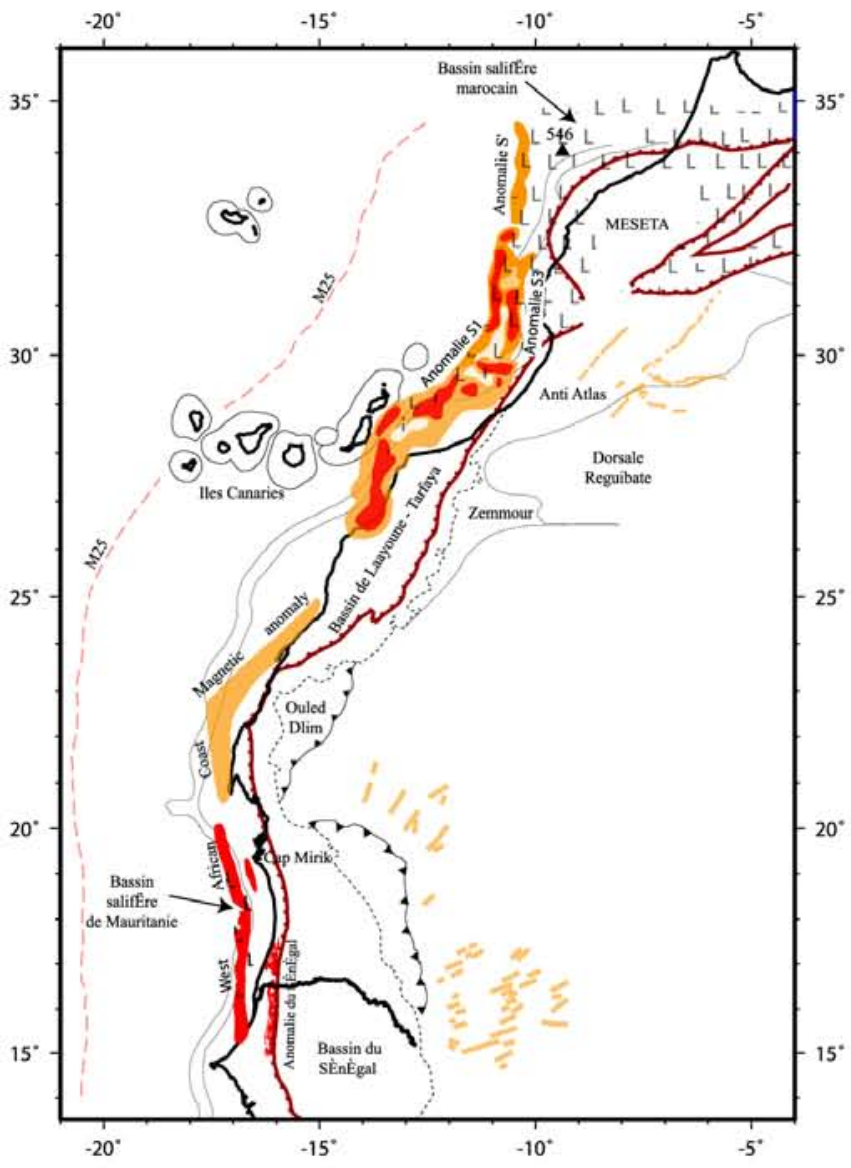

Figure 3 : Structures de la marge nord-ouest africaine (mêmes conventions que pour la fig.1). L'anomalie magnétique ouestafricaine (WACMA) se présente en trois segments : au Sud, l'anomalie continentale du Sénégal (d'après [16]) a été complétée par les données en mer (cf fig. 4); au Centre, le contour de l'anomalie provient également d'une compilation des données marines disponibles (le hiatus vers $200 \mathrm{~N}$ provient d'un manque de données); le segment septentrional (anomalies S1 et S3) est schématisé à partir de la carte de $[36,28]$. Noter le segment $S^{\prime}$ qui prolonge au Nord l'anomalie S1 et marque la limite des évaporites du bassin marocain profond. On a reporté sur la Meseta l'extension maximale probable de la halite au Lias Inférieur d'après [15]. Noter la présence d'un bassin à évaporites étroit à l'aplomb de la partie externe de la WACMA en regard de la Mauritanie. Les dykes représentatifs de la Province Magmatique de l'Atlantique Central (CAMP) sont indiqués d'après [1].

Figure 2: General map of the North-West African margin. Same legende as in Figure 1. The West African Coast Magnetic Anomaly (WACMA) can be divided in three segments: South, the continental magnetic anomaly of Senegal (after [16]) is completed by offshore-data; in the centre, the WACMA is drawn after all available magnetic profiles; north of $2600 \mathrm{~N}$, the WACMA (segment S1 eand S3) is drawn after the magnetic maps $[36,28]$. Note that the $S^{\prime}$ anomaly marks the limit of the salt basin. The maximun Moroccan salt basin is drawn after [15]. Note the tight salt basin straight over the extern limit of the WACMA, offshore of Mauritania. Dykes are from [1] and belongs to the Central Atlantic Magnetic Province (CAMP). 
l'ensemble constituait l'homologue de la ECMA (WACMA, pour "West African Coast Magnetic Anomaly). Klitgord et Schouten [14] ont par contre considéré que seul le segment continental du Sénégal était équivalent à la ECMA, l'anomalie S1 ayant l'âge de la BSMA, dont elle représenterait l'extension vers le Nord.

3.2 Une nouvelle interprétation de l'anomalie ouest-africaine

Une revue de tous les profils en mer disponibles (fig. 4) fait nettement apparaître au voisinage de la côte, entre $15^{\circ} \mathrm{N}$ et $20^{\circ} \mathrm{N}$, une anomalie magnétique parallèle à l'anomalie continentale du Sénégal. Nous proposons qu'il s'agisse là de l'anomalie marquant réellement le bord de la marge africaine et qu'elle complète la WACMA, L'anomalie continentale du Sénégal ne constitue plus alors qu'une partie de la WACMA, et l'anomalie ainsi reconstituée devient double, comme la ECMA dans le segment correspondant (fig.3 et 5). Les arguments en faveur de cette nouvelle identification tiennent en deux points : premièrement, l'homologie de forme avec la ECMA est meilleure, comme le montre la reconstruction réalisée; deuxièmement, la situation par rapport au bassin à évaporites de Mauritanie devient cohérente, comparable à celle du bassin de Caroline par rapport à la ECMA. Nous allons examiner ce point.

\subsection{Les bassins à évaporites}

Les évaporites de la marge africaine ont une répartition très voisine de celle de la marge américaine : le vaste bassin salifère du Maroc [38, 23], ouvert sur le Nord, se compare à celui de Nouvelle Ecosse par son extension et sa situation visà-vis des structures de la marge. Comme lui, il présente une terminaison étroite vers le Sud, comme lui il occupe une situation actuelle extrêmement profonde, puisqu'il se prolonge largement sous la plaine abyssale, à $8 \mathrm{~km}$ de profondeur. On n'a pas reporté, dans la littérature, d'évaporites dans le vaste bassin de Laâyoune-Tarfaya qui lui fait suite au Sud des Canaries. On retrouve par contre au large de la Mauritanie, sur $300 \mathrm{~km}$ de longueur, une étroite zone à manifestations diapiriques qui fait face à celle de Caroline sur la marge américaine. On connaît enfin, sur le plateau de Casamance, un autre bassin à évaporites (in [38]), sans correspondant du côté américain puisqu'il fait face au "Blake Plateau», une structure anormale de la marge (en relation avec

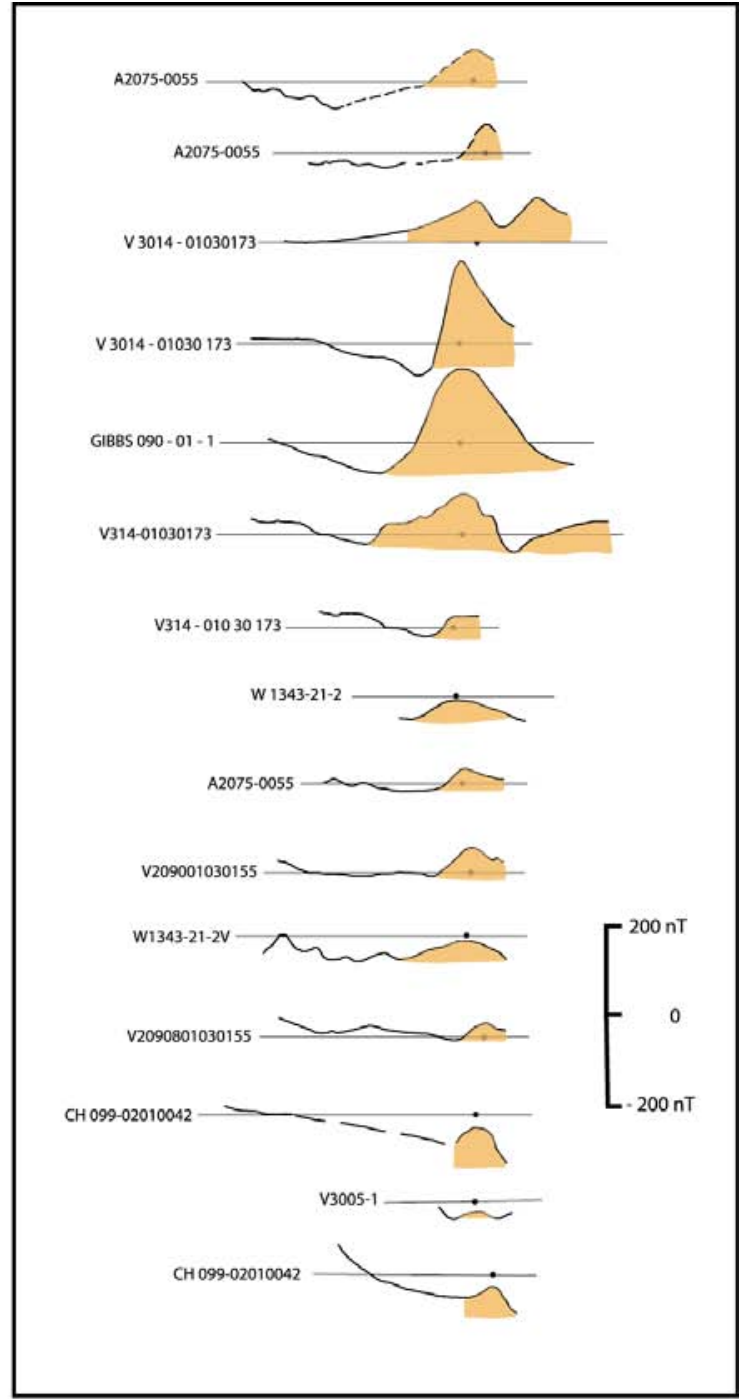

Figure 4 : Pointé de la partie occidentale de la WACMA à partir de tous les profils marins disponibles

entre $15^{\circ} \mathrm{N}$ et $20^{\circ} \mathrm{N}$.

Figure 4: All available magnetic profiles used for the determination of the WACMA between $15^{\circ} \mathrm{N}$ and $20^{\circ} \mathrm{N}$.

un point triple) mise en place au Dogger. Les évaporites du bassin nord marocain ont été atteintes par forage [40] et leur continuité avec les évaporites connues sur le pourtour de la Meseta et dans les sillons atlasiques a été clairement établie [26] : leur âge correspond sans doute possible à celui de la série ARGO américaine [10]. Le bassin marocain est délimité vers le large par l'anomalie S1 et par son prolongement $S$ ', ainsi que l'ont établi très tôt [6] et ainsi que le confirment les résultats de la campagne SISMAR $[[31,2]$. Sa position est donc similaire à celle du bassin de Nouvelle Ecosse vis-à-vis de la ECMA.

La fig. 6 illustre la parfaite homologie de forme des anomalies ECMA et S1. 


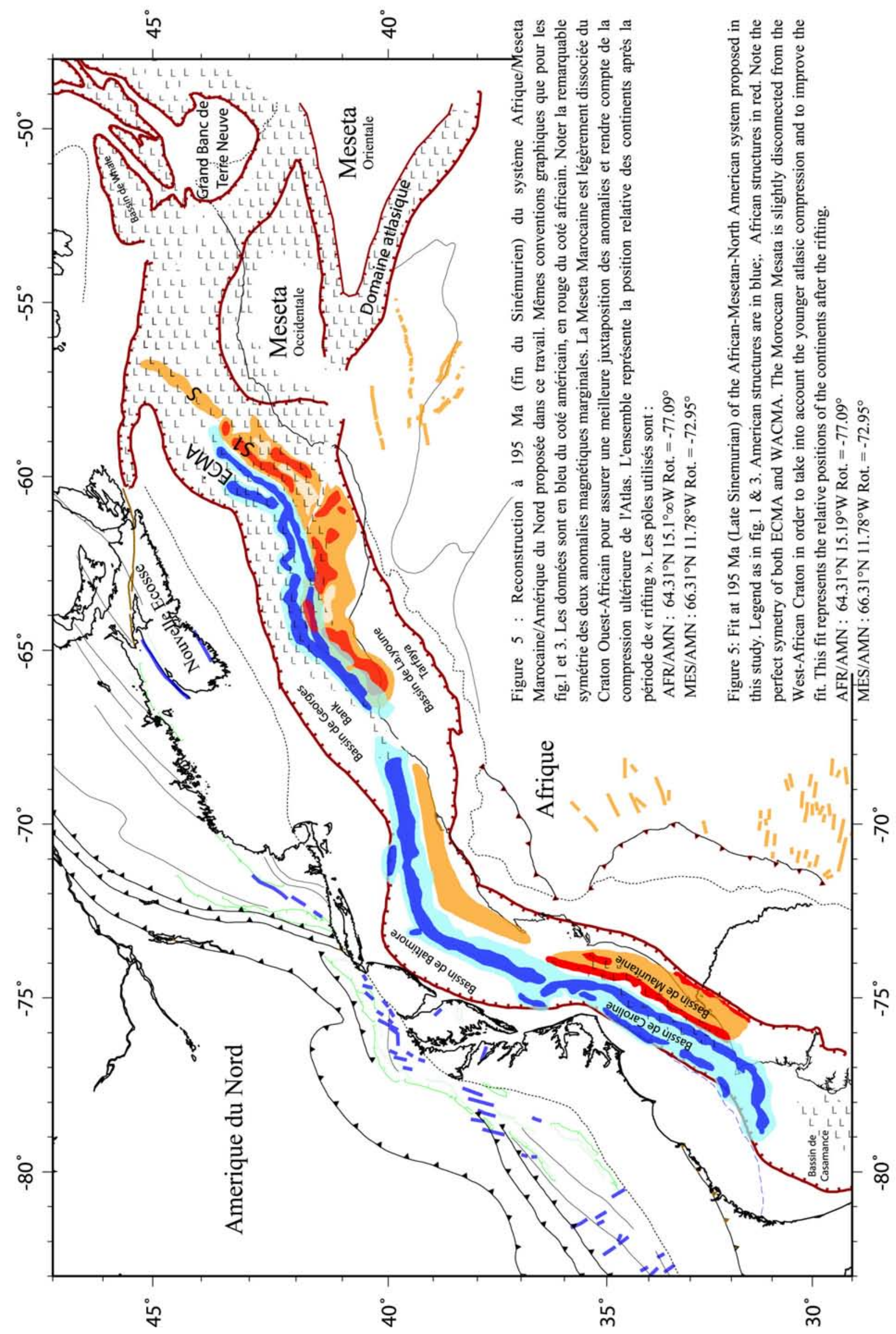


Par rapport à la reconstruction de la fig. 5 dont elle est dérivée, elle présente un stade légèrement postérieur, les bassins à évaporites étant séparés d'une centaine de $\mathrm{km}$ par ce qui représente sans doute la première croûte océanique atlantique, et peut-être la plus ancienne croûte océanique existant actuellement.

\section{Discussion}

La fig. 5 propose une reconstruction fondée sur la juxtaposition des deux anomalies magnétiques marginales, ECMA et WACMA. La Meseta marocaine a été légèrement disjointe du craton ouestafricain (voir aussi fig.6), ce qui permet un meilleur ajustement et rend compte de la compression postérieure du domaine atlasique.

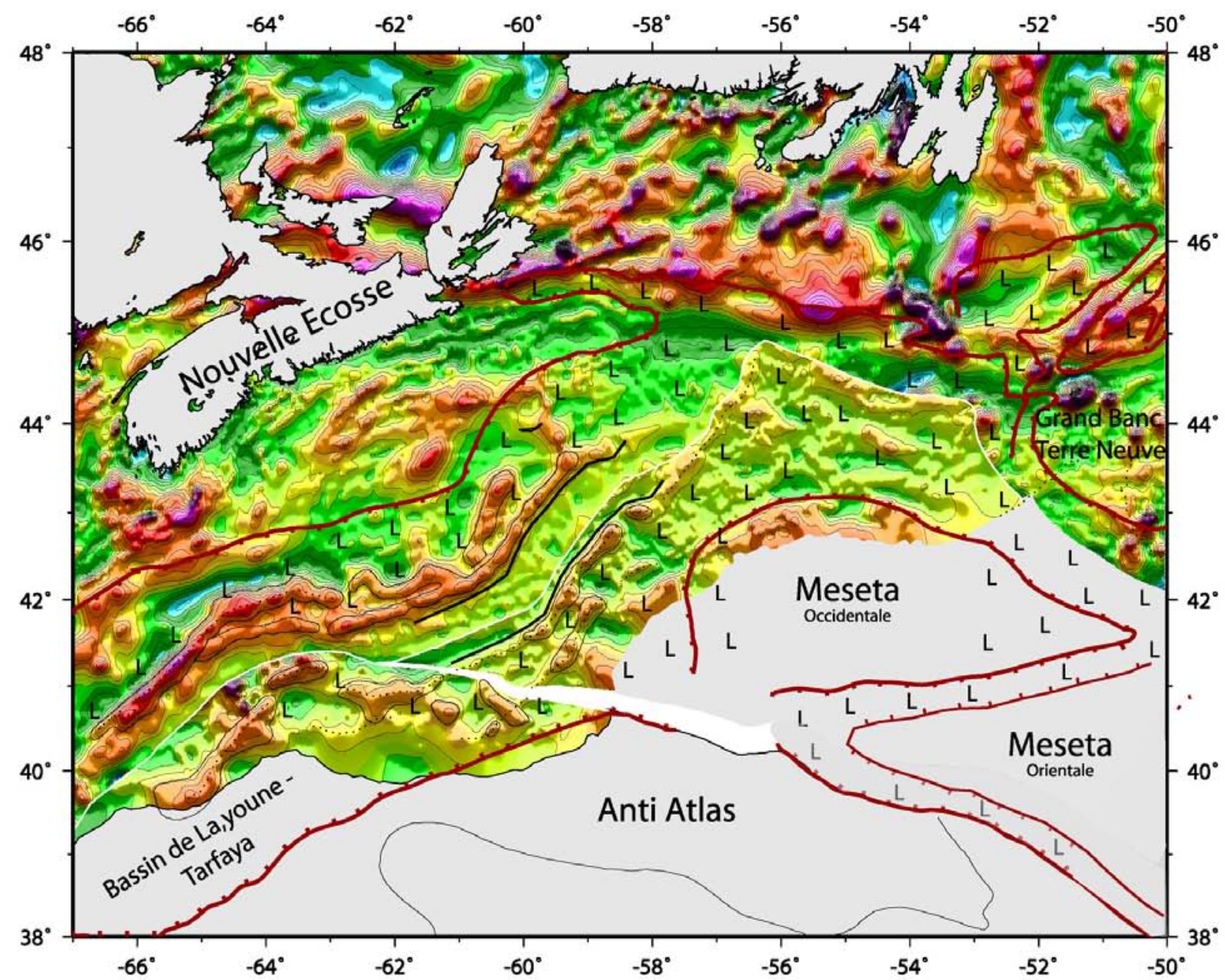

Figure 6 : Figure montrant la parfaite homologie de l'anomalie S1 avec la ECMA de Nouvelle Ecosse. L'anomalie S' n'a pas d'équivalent sur la marge américaine. Les données proviennent de la carte des anomalies magnétiques [36]. Le trait blanc matérialise la coupure entre les fichiers. La Meseta est déplacée par rapport à l'Afrique avec les mêmes paramètres que dans la fig. 5. Ils sont légèrement modifiés dans le sens d'une ouverture entre l'ensemble Afrique/Meseta et l'Amérique du Nord.

Les pôles utilisés sont :

AFR/AMN : $64.62^{\circ} \mathrm{N} 15.29^{\circ} \mathrm{W}$ Rot. $=-75.64^{\circ}$

MES/AMN : $66.69^{\circ} \mathrm{N} 11.82^{\circ} \mathrm{W}$ Rot. $=-71.52^{\circ}$

Figure 6: Slightly opened fit of the African-Mesetan-North American system, derived from the fit presented in figure 5. Note the perfect homology of the S1 anomaly and the Nova Scotian ECMA. S' anomaly does not have any american homologue. Magnetic data are from [36]. White lines show the separation between the plates. The Moroccan Meseta is slightly disconnected from the West-African Craton as in figure 5.

AFR/AMN : $64.62^{\circ} \mathrm{N} 15.29^{\circ} \mathrm{W}$ Rot. $=-75.64^{\circ}$

MES/AMN : $66.69^{\circ} \mathrm{N} 11.82^{\circ} \mathrm{W}$ Rot. $=-71.52^{\circ}$ 
Quel est l'âge du système ainsi reconstitué? L'âge Jurassique Moyen, souvent cité, ainsi que celui, plus précis, de $175 \mathrm{Ma}$, proviennent des reconstructions de Klitgord et Schouten [14]. Pour ces auteurs, et contrairement à ce que démontrent les fig 5 et 6 , l'anomalie S1 du Maroc ne correspondait pas à la ECMA de Nouvelle Ecosse, mais au prolongement vers le Nord de la BSMA datée du Callovien Inf. Pour dater la ECMA, les taux mesurés dans l'échelle de Kent et Gradstein entre l'anomalie M25 (156 Ma) et la BSMA (165 Ma), ont été simplement extrapolés jusqu'à sa limite. L'âge ainsi obtenu (175 Ma) a été largement adopté comme datant la fin du rifting et la première croûte océanique.

Notre reconstruction montre que 1'âge des anomalies magnétiques marginales doit être apprécié sur d'autres critères. Puisque les bassins américains et africains se trouvent précisément juxtaposés en même temps que sont juxtaposées les anomalies magnétiques marginales, la fin des évaporites (fin du Sinémurien, 195 Ma env. pour Odin et Odin [22] et Gradstein et al. [4]) peut dater le début de l'expansion océanique, postérieurement au stade correspondant à l'élaboration de ces anomalies marginales : on peut en effet penser qu'elle a une cause purement tectonique, l'accroissement de la tranche d'eau responsable du changement de faciès provenant d'une accélération de la subsidence liée au nouveau régime.

Un second critère se trouve dans l'âge des manifestations volcaniques. On sait que les dépôts de la fin du Trias et du début du Lias sont associés, très généralement, à d'importantes manifestations volcaniques $[20,1]$. Des deux côtés de l'Atlantique, on estime qu'un épisode volcanique majeur (Central Atlantic Magmatic Province : CAMP, [24]) se situe à la base du Lias, peu avant la fin de la formation à évaporites ou en coïncidence avec elle dans d'autres endroits $[39,15]$. Les travaux de datation les plus récents [18] tendent à situer l'essentiel de l'activité dans une période très restreinte autour de $200 \mathrm{Ma}$, avec un échelonnement des mesures entre $191 \mathrm{Ma}$ et 205 Ma qui corrobore les observations de terrain [9]. La reconstruction que nous proposons coïncide donc à peu de chose près avec ce pic d'activité à la fois tectonique et volcanique qui paraît bien marquer la vraie naissance de l'Atlantique.

\section{Conclusion}

Depuis plus de vingt ans, malgré un évident consensus sur toutes les observations majeures concernant la marge américaine et malgré la précision de celles-ci, une indécision s'est toujours manifestée lorsqu'il s'est agi de faire la part de l'évolution intra continentale (« rifting») et de l'évolution proprement océanique («drifting»), autrement dit, de les rapporter à un modèle précis de mouvement des plaques. Les essais de synthèse incluant la marge africaine n'ont pas levé cette indécision $[15,28]$.

L'âge le plus généralement admis pour la première croûte océanique est Jurassique Moyen, autour de 175 Ma. Mais les phénomènes que l'on s'accorde à rattacher à la période de «rifting » et à sa transition au «drifting », et en particulier l'âge de la fin des évaporites, sont d'un âge nettement plus ancien: fin du Sinémurien, soit quinze à vingt millions d'années plus tôt. Cette contradiction est matérialisée par les cartes d'extension des évaporites de la formation ARGO au large de la Nouvelle Ecosse qui impliquent pour la ECMA et la croûte océanique adjacente un âge encore plus ancien. Nous avons montré qu'à l'évidence, les évaporites ainsi cartographiées ne pouvaient être en place, et que la ECMA coïncidait avec la limite vraie de cette formation, tout comme l'anomalie S1 coïncide avec la limite du bassin à évaporites marocain. Nous avons montré aussi qu'une modification de l'interprétation de l'anomalie ouest africaine au large du Sénégal permettait tout à la fois un meilleur assemblage avec la ECMA et une meilleure intégration du bassin à évaporites, celui-ci se retrouvant dans une position de symétrie avec le bassin de Caroline qui lui fait face dans la reconstruction. Ainsi se trouve clarifiée une première question: la reconstruction qui ajuste comme nous l'avons fait les deux anomalies magnétiques caractéristiques des deux marges de l'Atlantique Central est en même temps celle qui permet de reconstituer, à son extension maximum, le bassin à évaporites du Lias Inférieur. L'anomalie BSMA, identifiée et datée du côté américain au Sud de $40^{\circ} \mathrm{N}$, correspond à un épisode de l'histoire déjà largement intra-océanique de l'Atlantique Central, plus de 30 millions d'années plus tard (au Callovien Inf). Cette longue phase initiale n'a toutefois créé qu'un océan de largeur réduite, (moins de $500 \mathrm{~km}$ ) ; elle a donc été extrêmement lente.

Cette solution ouvre la porte à une explication spécifique de l'activité tectonique et volcanique largement attestée au Jurassique Moyen, et qui doit être distinguée de l'activité liée au rifting proprement dit, à la fin du Trias et au Lias Inférieur. Au Jurassique Moyen, à partir du Callovien à coup sûr 
(BSMA), mais peut-être plus tôt (à partir de l'anom. E ?), la tectonique traduit l'accélération importante du mouvement de l'Afrique par rapport à l'Amérique et à l'Europe, le passage à des vitesses de plusieurs cm par an que l'on connaît dans les océans actuels ;

\section{References}

[1] H. Bertrand, M. Westphal, Comparaisons géologiques et paléomagnétiques des tholeïtes du Maroc et de la côte orientale de l'Amérique du Nord : implications pour l'ouverture de l'Atlantique, Bull. Soc. Géol. France, 7 XIX 3 (1977) 513 - 520.

[2] I. Contrucci, F. Klingelhöfer, J. Perrot, R. Bartolomé, M.A. Gutscher, M. Sahabi, J.A. Malod, J.P. Réhault, The Crustal Structure of the NW Moroccan Continental Margin from Wide-angle and Reflection Seismic Data, Geophys. J. Int., sous press.

[3] W.P. Dillon, P. Popenoe, The Blake Plateau and Carolina Trough, in : The Atlantic Continental Margin, R.E. Sheridan, J.A. Grow (Eds.), The Geological Society of America, The Geology of The North America, 1988, pp. 291-328.

[4] M.F. Gradstein, F.P. Agterberg, J.G. Ogg, J. Hardenbol, P. Van Veen, J. Thierry, Z. Huang, A Mesozoïc Time Scale, J. Geophys. Res., 99 B12 (1994) 24051-24074.

[5] D.E. Hayes, P.D. Rabinowitz, Mesozoic magnetic lineations and the magnetic Quiet Zone Off Northwest Africa, Earth Planet. Sci. Lett., 28 (1975) 105-115.

[6] K. Hinz, H. Dostmann, J. Fritsch, The continental margin of Morocco : seismic sequences, structural elements and the geological development, in : U. Von Rad, K. Hinz, M. Sarntheim, E. Seibold (Eds.), Geology of the North West African Continental Margin, Springer - Verlag, Berlin Heidelberg New York, 1982, pp. 34-60

[7] W.S. Holbrook, G.M. Purdy, R.E. Sheridan, L. Glover, M. Talwani, J. Ewing, D.R. Hutchinson, Seismic structure of the U.S. Mid-Atlantic continental margin, J. Geophys. Res., 99 B9 (1994) 17871-17891.

[8] W.T. Holser, G.P. Clement, L.F. Jansa, J.A. Wade, Evaporite deposits of the North Atlantic Rift, in : W. Manspeizer (Ed.), Triassic - Jurassic Rifting Continental Breakup and the origin of the Atlantic Ocean and Passive Margins, Elsevier, Amsterdam, 1988, pp. 525-553.

[9] S. Huon, J.J. Cornée, A. Piqué, N. Raïs, N. Clauer, N. Liewig, R. Zayane, Mise en évidence au Maroc d'événements thermiques d'âge triasico-liasique liés à l'ouverture de 1'Atlantique, Bull. Soc. Géol. France, t $164 \mathrm{n}^{\circ} 2(1993) 513-520$.

[10] L.F. Jansa, J. Wiedmann, Mesozoic-Cenozoic development of the Eastern North American and Northwest African continental margins : A comparison, in : U. Von Rad, K. Hinz, M. Sarnthein, E. Seibold (Eds), The Geology of the Northwest African Continental Margin, Springer-Verlag, Berlin Heidelberg New York, 1982, pp. 215-269.

[11] L.F. Jansa, J.P. Bujak, G.L. Williams, Upper triasic salt deposits of the western North Atlantic, Can. J. Earth Sci., 17 (1980) 547-559.

[12] C.E. Keen, D.P. Potter, Formation and evolution of the Nova Scotia rifted margin : evidence from deep seismic reflection data, Tectonics, 144 (1995) 918-932. elle marque, dans ce sens là, le véritable début de la cinématique des plaques mésozoïques.

D'un autre côté, il nous paraît que l'on peut maintenant, sur la base de ce que nous proposons, envisager une approche réellement quantitative de la période de « rifting ».

[13] M.J. Keen, C.L. Williams, Geology of the Continental Margin of Eastern Canada, The Geological Survey of Canada, Ottawa, 1990.

[14] K.D. Klitgord, H. Schouten, Plate kinematics of the Central Atlantic, in : P.R. Vogt, B.E. Tucholke (Eds.), The Western North Atlantic Region, The Geology of North America, The Geological Society of America, M, 1986, pp. 351-377.

[15] P. Le Roy, A. Piqué, Triassic-Liassic Western Moroccan synrift basins in relation to the Central Atlantic opening, Marine Geology, 172 (2001) 359381 .

[16] J.L. Liger, structure profonde du bassin sénégalomauritanien - interprétation des données gravimétriques et magnétiques, Trav. Lab. Sci. Terre, St Jérôme, Marseille,16, 1980.

[17] W. Manspeizer, Triassic - Jurassic Rifting Continental Breakup and the origin of the Atlantic Ocean and Passive Margins, Part A \& B, Elsevier, Amsterdam, 1988.

[18] A. Marzoli, P.R. Renne, E.M Piccirillo, M. Ernesto, G. Bellieni, A. DeMin, Extensive 200-Million-Year Old Continental Flood Basalts of the Central Atlantic Magmatic Province, Sciences, 284 (1999) 616-618.

[19] P. Matte, Variscides between the Appalachians and the Urals : Similarities and differences between Paleozoic subduction and collision belts, in : M., Catalan, J.R., Hatcher (Eds.), Variscan - Appalachian dynamics : The building of the late Paleozoic basment, The Geological Society of America, 364, Boulder, Colorado, 2002, pp. 239-251.

[20] P.R. May, Pattern of Triassic-Jurassic diabase dikes around the North Atlantic in the context of the predrift configuration of the continents, Geol. Soc. Am. Bull., 82 (1971) 1285-1292.

[21] McBride, K.D. Nelson, Integration of COCORP deep reflection and magnetic anomaly analysis in the southeastern United States : implications for origin of the Brunswick and East Coast Magnetic Anomalies, Geol. Soc. Amer. Bull., 100 (1988) 436-445.

[22] G.S. Odin, C. Odin, Echelle numérique des temps géologiques, Géochronique, 35 (1990) $12-21$.

[23] J.L. Olivet, J. Bonnin, P. Beuzart, J.M. Auzende, Cinématique de l'Atlantique Nord et Central, 108, CNEXO, Plouzané, 1984.

[24] P.E. Olsen, Giant Lava Flows, Mass Extinctions, and Mantle Plumes, Science, 284 (1999) 604-605.

[25] G. Pautot, J.M. Auzende, X. Le Pichon, Continous deep sea salt layer along North Atlantic Margins related to early phase of rifting, Nature 227 (1970) $351-354$.

[26] A. Piqué, Geology of Northwest Africa, Gebrüder Borntraeger, Berlin - Stuttgart, 2001.

[27] P.D. Rabinowitz, The Boundary between Oceanic and Continental Crust in The Western North Atlantic, in : The Geology of The Continental Margins, C.A. Burke, 
C.L. Drake (Eds.), Springer - Verlag, New York, 1974, pp. $67-84$

[28] H.A. Roeser, C. Steiner, B. Schreckenberger, M. Block, Structural development of the Jurassic Magnetic Quiet Zone off Morocco and identification of Middle Jurassic Magnetic Lineations, J. Geophys. Res., 107 B10 (2002).

[29] P.A. Rona, J. Brakle, J.R. Heirtzler, Magnetics anomalies in the Northeast Atlantic between the Canary and Cap Verde Islands, J. Geophys. Res., 7535 (1970) $7412-7420$.

[30] J. Roussel, J.L. Liger, A review of deep structure and ocean-continent transition in the Senegal basin (West Africa), Tectonophysics, 91 (1983) 183-211.

[31] M. Sahabi, L. Fidalgo, J.L. Olivet, D. Aslanian, L. Matias, M. Rabineau, M. Patriat, J.P. Réhault, J. Malod, M. Moulin, M. Bouabdelli, Evolution Mésozoïque de 1'Atlantique Central et problème de la reconstitution initiale : hypothèses et discussions, RST Nantes (2002) 209.

[32] R.E. Sheridan, D.L. Musser, L. Glover III, M. Talwani, J. Ewing, W.S. Holbrook, G.M. Purdy, R. Hawman, S. Smithson, Deep Seismic reflection data of EDGE U.S. Mid-Atlantic Continental Margin experiment : Implications for Appalachian sutures and Mesozoic rifting and magmatic underplating, Geology, 21 (1993) $563-567$.

[33] R.E. Scheridan, J.A. Grow, The Atlantic Continental Margin, The Geology of North America, The Geological Society of America, V I-2, Colorado, 1988.

[34] Sheridan, F.M. Gradstein, et al., Early history of the atlantic Ocean and gaz hydrate on the Blake Outer Ridge : results of the Deep - Sea Drilling Project Leg 76, Geol. Soc. Amer. Bull., 03 (1983) 876-885.

[35] E. Uchupi, K.O. Emery, C.O. Bowin, J.D. Phillips, Continental margin off Western Africa : Senegal to
Portugal, Amer. Assos. Petrol. Geol. Bull., 605 (1976) 809-878.

[36] J. Verhoef, W.R. Roest, R. Macnab, J. Arkani-Hamed, Membres of the Project Team, Magnetic anomalies of the Arctic and North Atlantic Oceans and Adjacent land areas, The Geological Survey of Canada, 1996.

[37] P.R. Vogt, C.J. Anderson, D.R. Bracey, Mesozoïc magnetic anomalies, sea floor spreading, and geomagnetic reversals in the southwestern North Atlantic, J. Geophys. Res., 7620 (1973) 4796-4823.

[38] U. Von Rad, K. Hinz, M. Sarntheim, E. Seibold, Geology of the North West African Continental Margin, Springer - Verlag, Berlin Heidelberg New York, 1982.

[39] J.A. Wade, B.C. MacLean, The Geology of the Southeastern margin of Canada, in : M.J. Keen, C.A. Williams (Eds.), Geology of Canada, Geology of the Continental Margin of Eastern Canada, Geological Survey of Canada, 1990, pp. 167-238.

[40] E.L. Winterer, K. Hinz, et al., The evolution of the Mazagan continental margin : a synthesis of geophysical and geological data with results of drilling during deep sea drilling project leg 79, in : J.H. Blakeslee (Ed), Initial Reports of DSDP, LXXIX, 1984, pp. $893-919$.

[41] G. Wissmann, H.A. Roeser, A magnetic and halocinetic structural pangaea fit of Northwest Africa and North America, Geol. Jb., E 23 (1982) 43-61.

[42] P.A. Ziegler, Post-hercynian plate reorganisation in the Tethys and Arctic - North Atlantic domains, in : W. Manspeizer (Ed.), Triassic - Jurassic rifting Continental Breakup and the Origin of the Atlantic Ocean and Passive Margins, Elsevier, Amsterdam, 1988, pp. 711-755. 